\title{
$O$ cotidiano de cuidadores informais de pacientes em tratamento quimioterápico ${ }^{1}$
}

\author{
Mariana Ortelani de Toledo ${ }^{\mathrm{a}}$, Maria Luisa Gazabim Simóes Ballarin ${ }^{\mathrm{b}}$ \\ ${ }^{a}$ Especialização em Residência em Terapia ocupacional em Contextos Hospitalares pela \\ Pontifícia Universidade Católica de Campinas - PUC Campinas, Terapeuta Ocupacional do \\ Centro de Referência do Jovem e Adolescente, Araraquara, SP, Brasil \\ 'Doutora em Saúde Mental, Universidade Estadual de Campinas - UNICAMP, \\ Docente da Faculdade de Terapia Ocupacional, Centro de Ciências da Vida, \\ Pontifícia Universidade Católica de Campinas - PUC, Campinas, SP, Brasil
}

\begin{abstract}
Resumo: Este trabalho tem por objetivo discorrer sobre o cotidiano de cuidadores informais - CIs de pacientes com câncer assistidos em uma unidade ambulatorial de quimioterapia de um hospital universitário do município de Campinas, estado de São Paulo. É parte de uma investigação mais ampla. O estudo foi aprovado por Comitê de Ética em Pesquisa sob o protocolo de $\mathrm{n}^{\circ} 0288 / 10$, sendo que todos os preceitos éticos foram adotados de acordo com o preconizado na resolução 196/96. Caracteriza-se como estudo de natureza descritiva, quantiqualitativo, em que se realizou pesquisa bibliográfica e de campo. O instrumento utilizado para a coleta de dados junto a 14 CIs foi a entrevista semiestruturada. Os dados obtidos foram analisados com base nos objetivos do estudo e nas formulações teóricas pertinentes à Teoria da Representação Social. Os resultados evidenciaram que 92,8\% dos CIs são do sexo feminino, $57,1 \%$ delas são casadas, com idade entre 41 e 60 anos $(78,5 \%)$, residentes na mesma moradia do familiar adoecido e cujo grau de parentesco correspondia a: filha-mãe, 35,7\%; seguido de esposa-marido, 21,4\%. Na percepção dos CIs, a função desempenhada interferiu em suas atividades cotidianas, restringindo atividades de lazer, autocuidado e trabalho. O estudo ampliou a compreensão sobre os impactos que a função de cuidar acarreta no cotidiano dos CIs investigados, contribuindo para fomentar as discussões acerca da necessidade de construção de intervenções e estratégias terapêuticas ocupacionais que facilitem o cotidiano desses CIs.
\end{abstract}

Palavras-chave: Cuidadores, Oncologia, Terapia Ocupacional.

\section{The daily life of informal caregivers of patients undergoing chemotherapy}

\begin{abstract}
This paper aims to discuss the daily life of Informal Caregivers (ICG) of cancer patients assisted in an outpatient chemotherapy unit of a university hospital in Campinas, São Paulo state. This study is part of a broader investigation. It was approved by a Research Ethics Committee under protocol n. 0288/10, and all ethical principles were adopted in accordance with the recommendations of Resolution 196/96. It is a descriptive, quantitative and qualitative study with literature and field research. Semi-structured interviews were conducted with 14 ICGs for data collection. The data were analyzed based on the goals of the study and the theory and practice pertaining to the Theory of Social Representation. The results showed that (92.8\%) of the ICGs are female $(57.1 \%)$, married, between 41 and 60 years old (78.5\%), living in the same household of the sickened family members at the following degrees of relatedness: (35.7\%) mother-daughter, followed by (21.4\%) husband-wife. In the Informal Caregivers' perception, their role interfered with their daily activities, bringing restrictions to leisure activities, self-care and work. The study expanded the understanding of the impacts that the function of caring brings to their everyday activities, helping to foster discussions about the need to implement actions and interventions of occupational therapy strategies that facilitate the daily lives of ICGs.
\end{abstract}

Keywords: Caregivers, Medical Oncology, Occupational Therapy.

Autor para correspondência: Mariana Ortelani de Toledo, Centro de Ciências da Vida, Faculdade de Terapia Ocupacional, Pontifícia Universidade Católica de Campinas, Av. John Boyd Dunlop, s/n, Jardim Ipaussurama, CEP 13059-900, Campinas,

SP, Brasil, e-mail: mlballarin@puc-campinas.edu.br

Recebido em 17/8/2011; Revisão em 4/4/2012; Aceito em 9/4/2012. 


\section{Introdução}

O surgimento de uma doença em um membro da família acarreta inúmeras alteraçôes na estrutura familiar, que vão além da esfera biológica. No caso de uma doença como o câncer é comum a pessoa precisar de ajuda contínua em função das limitaçôes decorrentes do próprio processo de adoecimento, o que a torna muitas vezes dependente de cuidado integral. Nesse contexto salienta-se que as contribuiçốes da Antropologia da Saúde têm demonstrado que o enfrentamento de um processo de adoecimento está diretamente relacionado à cultura, aos valores, às crenças e às experiências pessoais. Ao interagirem entre si, todos esses aspectos fornecem uma estrutura de referência para a compreensão da enfermidade. Segundo Queiroz (2003), parece haver um consenso nas Ciências Sociais de que a compreensão da saúde e da doença não pode prescindir de fatores sociais, culturais e psicológicos, pois esse binômio depende de componentes subjetivos e emocionais relacionados à experiência de vida.

Ao figurar no grupo de doenças que trazem grande impacto psicológico e social, o câncer é, ainda, considerado estigmatizante e carregado de negativismo (ZAGO et al., 2001; MELO et al., 2005). Tal termo é utilizado para designar um conjunto de mais de 200 doenças que têm em comum o crescimento desordenado (maligno) de células que invadem os tecidos e órgãos, podendo espalhar-se, na forma de metástase, para outras regióes do corpo. Dividindo-se rapidamente, essas células tendem a ser muito agressivas e incontroláveis, determinando a formação de tumores ou neoplasias malignas (INSTITUTO..., 2004; PALM, 2007; DE CARLO; QUEIROZ; SANTOS, 2008; SALES et al., 2010).

Estimativas apontam para o surgimento de 489.270 novos casos de câncer no Brasil em 2011 (INSTITUTO..., 2009; SILVA et al., 2010). Em 2005 foram registrados pelo Sistema Único de Saúde - SUS 423 mil internaçôes por neoplasias malignas (câncer) e 1,6 milhão de consultas ambulatoriais em Oncologia. Nesse mesmo ano, o Ministério da Saúde instituiu através da Portaria GM 2.439/05 a Política Nacional de Atenção Oncológica - PNAO, que direciona e define como focos de atuação a promoçáo, a prevenção, o diagnóstico, o tratamento, a reabilitação e os cuidados paliativos.

No contexto de cuidados dedicados aos pacientes com câncer, além dos serviços e profissionais de saúde, emerge a figura do cuidador informal - CI, geralmente um membro da família que passa a assumir múltiplas funções, acompanhando o paciente na evoluçáo de sua doença em todas as suas fases (MACHADO; FREITAS;
JORGE, 2007). No presente estudo, o CI foi definido como o principal responsável pelo paciente, por prover os cuidados requeridos por ele e não possuir formação especializada para exercer a função de cuidador. Essa função muitas vezes implica não somente em cuidar do doente mas também em conciliar os cuidados à organização e gerenciamento das atividades e afazeres domésticas, exigindo que o $\mathrm{CI}$ reorganize a rotina de atividades diárias e seu cotidiano (PEDRO; MARCON, 2007). Tal aspecto mostra-se relevante, particularmente para a Terapia Ocupacional, que se caracteriza como uma área de conhecimento e uma prática de saúde que tem como objeto de conhecimento e intervençáo os problemas do homem em sua vida cotidiana de atividades. Assim, neste estudo buscamos compreender e discutir como o cuidar de uma pessoa com câncer e em tratamento quimioterápico pode afetar o cotidiano daquele que cuida.

\section{Considerações metodológicas}

Trata-se de um estudo do tipo descritivo exploratório, de natureza qualitativa, cujo desenvolvimento envolveu a realização de pesquisa bibliográfica e de campo. A pesquisa bibliográfica se deu a partir do levantamento e da revisão bibliográfica efetivados nas bases de dados LILACS e Scielo. Já a pesquisa de campo contemplou realizaçáo de entrevistas semiestruturadas junto a CIs de pacientes em tratamento quimioterápico, atendidos em hospital universitário do município de Campinas, estado de São Paulo.

As entrevistas foram realizadas individualmente, foram gravadas, transcritas e seguiram um roteiro previamente estabelecido, que objetivou apreender aspectos da rotina de vida diária e do cotidiano dos sujeitos investigados, bem como dados relacionados ao perfil sociodemográfico tais como: estado civil, número de filhos, ocupação, grau de escolaridade, grau de parentesco com o paciente, se reside com a pessoa que cuida.

Participaram do estudo 14 CIs selecionados por critério de conveniência, a partir de indicaçáo da equipe técnica de profissionais que atendiam os pacientes em tratamento quimioterápico e conheciam os CIs que os acompanhavam ao hospital.

O estudo foi aprovado por Comitê de Ética em Pesquisa, sob protocolo de no $0288 / 10$. Todos os participantes foram informados quanto aos objetivos do estudo e assinaram o Termo de Consentimento Livre e Esclarecido - TCLE, de acordo com os preceitos éticos previstos na Resoluçâo 196/96 (BRASIL, 1996). 
Os dados foram coletados entre os meses de maio e agosto de 2010, sendo que a pesquisadora frequentou o setor de quimioterapia sistematicamente, 2 a 3 vezes por semana. Algumas dificuldades foram observadas na etapa de realização das entrevistas, na medida em que a presença do CI no serviço estava condicionada à presença do paciente. Assim, dependendo da condição clínica do paciente, havia necessidade de novo agendamento para realizar a entrevista com o CI.

O caminho escolhido para analisar os dados obtidos foi refletir sobre o conteúdo do discurso, de modo a agrupá-los em categorias temáticas, considerando os objetivos estabelecidos e os referenciais teóricos pertinentes à teoria da representação social - RS. Entendemos que a teoria da representação social é uma importante ferramenta de análise da realidade social, já que possibilita enxergar as concepçóes que os diferentes grupos constroem a respeito do mundo e da realidade vivenciada. Segundo Spink (2004), a RS possui um caráter produtor de imagens significativas que expressam o trabalho do psiquismo humano sobre o mundo. Dito de outro modo, é o espaço do sujeito social lutando para dar sentido, interpretar e construir o mundo em que ele se encontra, ou seja, sua realidade. Por isso mesmo, implica em entender, interpretar e compreender os sentidos e as significaçôes que uma pessoa dá aos fenômenos em foco, por meio de técnicas de observação ampla e entrevistas com profundidade (TURATO, 2003). Ainda, de acordo com Minayo (1999), as representaçôes sociais sobre o processo saúde-doença determinam condutas terapêuticas concretas em cada grupo social, as quais são condizentes com a visão de mundo e de homem, sendo relevante o exame das práticas populares, as experiências do dia a dia e o senso comum.

\section{Resultados e discussão}

Do vasto material obtido a partir das entrevistas realizadas junto aos CIs resultaram discussôes relevantes. Nesse trabalho abordaremos especificamente o perfil sociodemográfico dos CIs, bem como algumas características dos pacientes em tratamento quimioterápico e o cotidiano dos CIs.

\section{Caracterização do perfil sociodemográfico dos CIs investigados}

Quanto às variáveis relacionadas ao perfil sociodemográfico dos CIs, os resultados obtidos relativos ao gênero e ao grau de parentesco evidenciaram que $92,8 \%$ eram do sexo feminino, sendo que $57,1 \%$ eram casadas, o grau de parentesco correspondia a filha-mãe, $35,7 \%$, seguido de esposamarido, 21,4\%. Esses resultados coincidem com os descritos nos estudos de Martins, Ribeiro e Garrett (2003), Py (2004) e Wanderbroocke (2002, 2005).

Araújo et al. (2009) atribuem esses achados ao fato de a mulher ter assumido historicamente a função de cuidadora dos filhos, dos pais, da família, confirmando assim nossos resultados. A autora salienta ainda que tal fato, também, é identificado na escolha das profissóes, tais como enfermagem, serviço social, entre outras, pois essas são marcadamente relacionadas ao cuidar.

Em relação à faixa etária observou-se que 57,1\% dos CIs apresentavam idade que variava entre $41 \mathrm{e}$ 60 anos, adultos em idade laborativa, dados similares aos descritos no estudo de Chaves e Vasconcelos (2006). No entanto, faz-se necessário destacar que neste estudo $79 \%$ dos CIs náo estavam exercendo atividade remunerada ou de trabalho.

O nível de escolaridade dos CIs entrevistados se mostrou diversificado, sendo que $7 \%$ dos $\mathrm{CIs}$ entrevistados não eram alfabetizados, $28,5 \%$ deles não haviam completado o ensino fundamental $\mathrm{e}$ $14,2 \%$ não tinham completado o ensino médio, somente $7 \%$ deles tinham instrução de nível superior. Constatou-se ainda que 78,5\% dos CIs residiam na mesma moradia do familiar adoecido, seguidos de $21,4 \%$ que, embora náo residissem no mesmo local, moravam próximos ao familiar que necessitava de cuidados e acompanhamento.

De modo geral, os resultados obtidos, em relaçáo às variáveis decritas, não diferem significativamente de outros estudos (GARRIDO; MENEZES, 2004; ALMEIDA, 2005).

\section{Considerações sobre os pacientes assistidos pelos CIs}

Os dados obtidos demonstraram que os CIs dedicavam-se ao cuidado de pacientes que apresentavam diferentes diagnósticos de câncer. Sendo que $57 \%$ eram pacientes do sexo feminino e $43 \%$ do masculino. Entre os pacientes do sexo feminino, $37 \%$ apresentavam câncer de mama, seguidos de linfoma. Já nos pacientes de sexo masculino, 66\%) apresentavam câncer de próstata. Foram identificados ainda pacientes com câncer de esôfago e intestino, cabeça e pescoço de ambos os sexos. Os resultados encontrados neste estudo estáo de acordo com os descritos no estudo de Guerra, Gallo e Mendonça (2005), cujos dados apontam para o fato de que no 
Brasil os tipos de câncer mais frequentes na população masculina são os de próstata, pulmão, estômago, cólon e reto e esôfago e, na populaçáo feminina, o de mama, seguido pelos cânceres de colo uterino, cólon e reto, pulmão e estômago.

Nas últimas décadas observa-se que a incidência de câncer de boca e de faringe tem aumentado, acompanhando o aumento no consumo de tabaco e de bebidas alcoólicas.

Ainda em relação aos pacientes assistidos pelos CIs, esses possuía idade que variava de 15 a 76 anos, sendo que $57 \%$ encontrava-se na faixa etária entre 55 e 76 anos. Salienta-se que os pacientes encontravam-se em diferentes fases de tratamento e estágio da doença, tendo se constatado casos de recidiva e, portanto, situaçóes que envolviam, por parte dos CIs, cuidados e atenção intensivos.

\section{Cotidiano dos CIs e o processo de cuidar do outro}

De acordo com Ballarin, Carvalho e Ferigato (2009), o ato de cuidar pressupóe do cuidador, uma postura ativa que permita reconhecer o outro na sua liberdade, na sua dignidade e singularidade. Portanto, salienta-se que a relaçáo de cuidado é dinâmica e se estabelece a partir de um contexto que vai se construindo processualmente a partir da convivência entre cuidador e pessoa que necessita de cuidados, a partir das vivências cotidianas. Nesse sentido e em relação ao ato de cuidar, a análise do material obtido junto aos entrevistados, evidencia que muitos cuidadores abdicaram de suas atividades rotineiras, deixando sua vida de lado para se dedicar à função de cuidar, conforme se observa nos relatos que se seguem:

[...] nada eu faço para mim, além de tomar banho e me alimentar, pouca coisa eu faço para mim [...] eu deixei de fazer muita coisa, muita coisa que en gostava de fazer [...] en fazia caminhada, eu trabalhava, ai, depois que ele ficon doente en tenho até medo de voltar a trabalhar e deixar ele (CI 14).

Eu deixei de pintar o cabelo, fazer minha unha [...] deixei também de ir a igreja (CI 2).

Minha vida mudou bastante. Eu costumava ir ao culto e depois sair com os amigos e hoje eu não consigo mais fazer isso (CI 4).

Os relatos dos CIs 14, 2 e 4 descritos acima apontam que as atividades de autocuidado (alimentação, higiene e cuidados pessoais) e as de caratér social e/ou lazer (sair com amigos) foram alteradas de modo significativo. De fato, segundo Gonçalves et al. (2006), Inocenti, Rodrigues e Miasso (2009) e Sales et al. (2010), a rotina do cuidador é diretamente influenciada pela demanda de cuidados e as necessidades do paciente, o que, consequentemente, pode privar o cuidador de sua sociabilidade cotidiana e interromper o curso normal da vida.

Ainda em relação ao cotidiano e a rotina de atividades dos CI constatou-se que desde que iniciaram a função de cuidador, o trabalho (atividade remunerada) foi a atividade que apresentou as maiores alteraçôes, conforme relatos:

[...] eu trabalhava, então a maioria do tempo eu ficava mais fora, ai, depois que eu comecei a cuidar dela, fico 24 horas por dia dentro de casa. [...] isso mudou muito. [...] Mas en queria não ficar só em casa, tanto só em casa [...] $\mathrm{Na}$ verdade o meu tempo é todo dela, todinho (CI 4).

[...] eu tive de me afastar 2 meses do trabalho, quando descobri que ele estava com câncer e começou o tratamento de quimioterapia. Depois, com o tratamento, ele começou a melhorar e eu voltei ao trabalho. Hoje estou aposentada (CI 13).

Gostaria de voltar a trabalhar, mas estou esperando a situação dela melhorar ou ficar estabilizada para procurar um novo emprego (CI 10).

Seja a partir de uma condição de afastamento total em relação ao trabalho remunerado ou na condição de afastamento temporário, o fato em si traz impactos importantes, tanto para a vida do CI, como para a vida dos familiares. Tal condição afeta os papéis sociais, ocupacionais e pessoais desempenhados pelo CI. Floriani (2004) e Floriani e Schramm (2006) descrevem que a sobrecarga econômica advinda da necessidade de deixar a atividade de trabalhar para cuidar de um paciente dependente caracteriza-se como aspecto estressor para o cuidador e sua família.

A idéia de sobrecarga é bastante ampla. Traduzido da língua inglesa, o termo sobrecarga do cuidador (caregiver burden) designa o estresse e se caracteriza como uma reação negativa aguda à prestação de cuidados. Emerge quando novas demandas de cuidados são introduzidas ou se intensificam e os cuidadores apresentam dificuldades para se adaptar ou modificar sua estratégia para atendê-las, vivenciando, assim, sobrecarga. O estresse relacionado aos cuidados é um fenômeno multidimensional que engloba quatro domínios, quais sejam: o quadro de cuidado (inclui as características do cuidador, o tipo de relacionamento estabelecido e o apoio social); as situaçóes de estresse relacionadas ao cuidado; os fatores que funcionam como moderadores da 
percepção de estresse (estratégias de enfrentamento); e as consequências dos cuidados sobre o bem-estar geral do cuidador (PAPASTAVROU et al., 2007).

Frente às exigências e demandas que a função impóe em relação a alguns aspectos da modificação de sua rotina diária, vêem-se desejosos e necessitados de compartilharem essa função com mais pessoas e/ ou até mesmo terem a possibilidade de contar com alguns momentos dedicados a si próprios. Os relatos que se seguem ilustram esse aspecto:

Seria bom se eu tivesse os finais de semana livres e pudesse dividir essa função com alguém. Mas não é facil (CI 8).

A gente em casa está vivendo pela E. [...] hoje eu estou assim, estou mais nervosa, irritada, qualquer coisa começo a chorar [...] não tenho paciência com ninguém, só tenho paciência com ela (CI 7).

A literatura tem demonstrado que a sobrecarga fisica, psíquica e social do cuidador restringe as atividades, traz preocupações, insegurança e isolamento, colocando-o diante de fatores que apresentam potencial para aumentar o risco de cansaço e estresse. Um número variado de perturbaçóes como depressão, medo, distúrbios do sono, rupturas de vínculos, entre outros, vêm sendo descritas, de acordo com Floriani e Schramm (2006).

Rezende et al. (2005) afirmam ser considerável a proporção dos cuidadores informais de pacientes com câncer que apresentam ansiedade e depressão. Existe interrelacionamento entre o sofrimento psíquico da família e o sofrimento do paciente, com consequências diretas sobre ambos. A depressão e a ansiedade do cuidador podem desencadear depressáo e ansiedade no paciente e vice-versa; ou seja, se a família é afetada pela doença, as reaçôes familiares também afetam o paciente. Tais evidências reforçam a ideia de que o processo de cuidar é bastante complexo, influenciando e sendo influenciado pelo contexto do adoecer e do morrer. Assim, é justificável a preocupação crescente em estudar esse impacto. Avaliar e tratar a ansiedade e a depressão no cuidador informal têm importante valor preventivo.Tal contexto reflete a importância e a necessidade dos familiares cuidadores receberem suporte psicológico e emocional adequados, para que se sintam preparados nesse processo (FERREIRA; SOUZA; STUCHI, 2008).

Nas situaçôes relacionadas ao ato de cuidar vivenciadas pelos cuidadores constatou-se que também emergiram ideias que expressavam resignação quanto aos limites que a função de cuidar impunha a sua própria rotina diária. Observou-se que tais ideias funcionam como determinantes da forma como cuidadores e familiares enfrentam o processo, conforme se pode depreender dos relatos que se seguem:
Neste momento estou me dedicando a ele, mas tenho certeza que isso é temporário, quando ele melhorar terei tempo e disposição para retornar as coisas que fazia [...] A gente se mobiliza para oferecer as melhores condiçōes para ele. A gente tem de aceitar [...] organizei uma novena aqui em casa para que eu e ele pudéssemos frequentar (CI 9).

A gente em casa tá assim [...] realizando os sonhos dela [...] ela se apoia muito em mim, acha que eu sou o porto seguro dela, mas não, ela é que é o anjo da minha vida, com ela eu aprendi muito (CI 7).

A gente chorou muito, eu falei para ele [paciente] assim, só que agora não vamos chorar mais, nós vamos à luta e seja o que Deus quiser (CI 13).

Segundo Pereira e Dias (2007), são inúmeras e diferentes as formas que os cuidadores e familiares encontram para o enfrentamento da doença e do processo que estão vivenciando. De fato, de acordo com Boff (1999), a atitude de cuidado provoca inquietaçóes, preocupaçóes e um certo sentido de responsabilidade. Assim, "a pessoa que tem cuidado se sente envolvida e afetivamente ligada ao outro" (BOFF, 1999, p. 92). Desse modo, tanto o choro como a religiosidade podem funcionar como alívio nos momentos difíceis, fornecendo consolo e fortalecimento.

Nesse sentido, a religiosidade ou espiritualidade nas situaçôes existenciais de sofrimento intenso assumem um lugar importante na vida dos indivíduos, uma vez que podem auxiliar os indivíduos a significarem esses eventos considerados sem sentido. Nas palavras da autora: "a fé e a religiosidade podem vir a preencher esse vazio explicativo para a doença que se instala, para a morte que se avizinha ou que já aconteceu" (VALLE, 2004, p. 30).

Kovács (2007) chama a atenção para o fato de que as situações que envolvem muito sofrimento, entre as quais o adoecimento, podem provocar mudaças significativas na vida, incluindo aspectos relacionados à espiritualidade, compreendida aqui como a busca pela compreensão do sentido da vida. Há, portanto, que se admitir pontos de intersecção e complementaridade entre a saúde mental e a espiritualidade, o que deve implicar na busca, por parte da equipe, de cuidados e de formas mais efetivas para intervir junto às famílias e aos pacientes considerando esta dimensão.

Os aspectos descritos a partir dos relatos evidenciam que os cuidadores, ao se envolverem com o ato de cuidar de seus familiares, passam a incorporar mudanças no cotidiano e nas atividades e tarefas de vida diária. Tais mudanças os tornam 
mais vulneráveis ao desgaste físico e emocional. Sendo assim, a OMS considera o atendimento às necessidades dos cuidadores um dos principais objetivos dos programas de cuidados paliativos.

É de fundamental importância que os profissionais de saúde mantenham-se atentos às necessidades de seus clientes, assistindo não só o indivíduo enfermo mas também seus familiares, para que eles não sejam sobrecarregados e possam manter uma vida de qualidade e proporcionar cuidados oportunos ao familiar que acompanham.

No âmbito do suporte necessário aos cuidadores, é possível diferenciar dois tipos: aqueles relacionados aos sistemas informais de suporte, explicados como redes de relacionamento entre membros da família, amigos e vizinhos que oferecem apoio e valorização do ato de cuidar; e o suporte social formal, relacionado a hospitais, atendimento domiciliar, instituiçôes da comunidade e de abrigamento e programas formais de capacitação de pessoal (GRATÃO, 2006).

De toda forma, entendemos que conhecer o modo e a percepção dos CIs em relação à sua qualidade de vida e ao cotidiano de cuidados que vivenciam pode favorecer a formulação de estratégias que objetivem facilitar o enfrentamento de todo o processo vivenciado. Dentro dessa perspectiva, Inocenti, Rodrigues e Miasso (2009) reafirmam a preocupação com o desenvolvimento de práticas e intervenções por parte dos profissionais de saúde que atendam as dimensôes de qualidade de vida. Essas devem reunir-se em torno de uma significação inclusiva, flexível, que valorize a opiniāo e o conhecimento do próprio $\mathrm{CI}$, de maneira que possam se sentir aceitos e compreendidos em suas dificuldades e limitaçôes.

\section{Considerações finais}

Os resultados do estudo evidenciaram que os CIs investigados eram prioritariamente do sexo feminino, casadas, residentes na mesma moradia do pacientefamiliar que acompanhavam, com idade entre 41 e 60 anos, que o grau de parentesco correspondeu majoritariamente a filha-mãe e esposa-marido e que, ao se dedicarem ao acompanhamento e cuidados de seus familiares adoecidos, os CIs vivenciavam um cotidiano permeado por uma rotina que as levara a abandonar atividades que exerciam anteriormete à função de cuidar, a exemplo do trabalho remunerado, e atividades de lazer e de caráter social, ficando assim vulneráveis a um ambiente estressor com sobrecarga de tarefas.

O estudo permitiu ampliar a compreensão sobre os impactos causados no cotidiano dos CIs explicitando a importância, por parte dos profissionais de saúde, de implementarem estratégias e intervençóes não somente direcionadas aos pacientes, mas também aos CIs.

\section{Referências}

ARAÚJO, L. Z. S. et al. Cuidador principal de paciente oncológico fora de possibilidade de cura, repercussōes deste encargo. Revista Brasileira de Enfermagem, Brasília, v. 62, n. 1, p. 32-37, jan./fev. 2009. http://dx.doi.org/10.1590/ S0034-71672009000100005

BALLARIN, M. L. G. S.; CARVALHO, F. B.; FERIGATO, S. H. Os diferentes sentidos do cuidado: consideraçōes sobre a atenção em saúde mental. $O$ Mundo da Saúde, São Paulo, v. 33, n. 2, p. 218-224, 2009.

BRASIL. Ministério da Saúde. Conselho Nacional de Saúde. Comissão Nacional de ética em Pesquisa. Resolução $\mathrm{n}^{\circ} 196$, de 10 de outubro de 1996. Diretrizes e normas regulamentadoras de pesquisa em seres humanos. Diário Oficial da República Federativa do Brasil, Brasília, DF, 16 out. 1996. Seção 1, n. 201.

BOFF, L. Saber cuidar: ética do humano - compaixão pela terra. Petrópolis: Vozes, 1999.

CHAVES, A. R. M.; VASCONCELOS, A. M. O cuidador no provimento do cuidado ao paciente com câncer avançado no Instituto Nacional de Câncer. Revista Brasileira de Cancerologia, Rio de Janeiro, v. 52, n. 1, p. 111, 2006.

DE CARLO, M. R. P.; QUEIROZ, M. E. G.; SANTOS, W. A. Terapia Ocupacional em Dor e Cuidados Paliativos - Princípios, Modelos de Intervenção e Perspectivas. In: DE CARLO, M. R. P.; QUEIROZ, M. E. G. Dor e Cuidados Paliativos: Terapia Ocupacional e Interdisciplinaridade. São Paulo: Editora Roca, 2008. p. 126-145.

FERREIRA, N. M. L. A.; SOUZA, C. L. B.; STUCHI, Z. Cuidados paliativos e família. Revista de Ciências Médicas, Campinas, v. 17, n. 1, p. 33-42, 2008.

FLORIANI, C. A. Cuidador familiar: sobrecarga e proteçáo. Revista Brasileira de Cancerologia, Rio de Janeiro, v. 50, n. 4, p. 341-345, 2004.

FLORIANI, C. A.; SCHRAMM, F. R. Cuidador do idoso com câncer avançado: um ator vulnerado. Cadernos de Saúde Pública, Rio de Janeiro, v. 22, n. 3, p. 527-534, 2006.

GARRIDO, R.; MENEZES, P. R. Impacto em cuidadores de idosos com demência atendidos em um serviço psicogeriátrico. Revista de Saúde Pública, São Paulo, v. 38, n. 6, p. 835-841, dez. 2004. http://dx.doi. org/10.1590/S0034-89102004000600012

GONÇALVES, L. H. T. et al. Perfil da família cuidadora de idoso doente/fragilizado do contexto sociocultural de Florianópolis, SC. Texto \& Contexto Enfermagem, Florianópolis, v. 15, n. 4, p. 570-577, jan./mar. 2006. http://dx.doi.org/10.1590/S0104-07072007000100026 GRATÃO, A. C. M. Demanda do cuidador familiar com idoso demenciado. 2006. 89 f. Dissertação (Mestrado em Enfermagem Fundamental)-Escola de Enfermagem de Ribeirăo Preto, Universidade de São Paulo, Ribeirão Preto, 2006.

GUERRA, M. L.; GALLO, C. V. M.; MENDONÇA, G. A. Risco de câncer no Brasil: tendências e estudos 
epidemiológicos mais recentes. Revista Brasileira de Cancerologia, Rio de Janeiro, v. 5, n. 3, p. 227-234, 2005.

INSTITUTO NACIONAL DO CÂNCER - INCA. Estimativa 2005: Incidência de câncer no Brasil. Rio de Janeiro: INCA, 2004.

INSTITUTO NACIONAL DE CÂNCER - INCA. Estimativa 2010: incidência de câncer no Brasil. Rio de Janeiro: INCA, 2009.

INOCENTI, A.; RODRIGUES, I. G.; MIASSO, A. I. Vivências e sentimentos do cuidador familiar do paciente oncológico em cuidados paliativos. Revista Eletrônica de Enfermagem, Goiânia, v. 11, n. 4, p. 858-865, 2009.

KOVÁCS, M. J. Espiritualidade e psicologia: cuidados compartilhados. O Mundo da Saúde, São Paulo, v. 31, n. 2, p. 246-255, 2007.

MACHADO, A. L. G.; FREITAS, C. H. A.; JORGE, M. S. B. O fazer do cuidador familiar: significados e crenças. Revista Brasileira de Enfermagem, Brasília, v. 60, n. 5, p.530-534, set./out. 2007. http://dx.doi.org/10.1590/ S0034-71672007000500009

MARTINS, T.; RIBEIRO, J. P.; GARRET, C. Estudo de validação do questionário de avaliação da sobrecarga para cuidadores informais. Psicologia, Saúde e Doenças, Lisboa, v. 4, n. 1, p. 131-148, 2003.

MELO, M. C. B. et al. A família e o processo de adoecer de câncer bucal. Psicologia em Estudo, Maringá, v. 10, n. 3, p. 413-419, 2005.

MINAYO, M. C. S. Ciência, técnica e arte: o desafio da Pesquisa Social. In: MINAYO, M. C. S. (Org.). Pesquisa social: teoria, método e criatividade. Petrópolis: Ed. Vozes, 1999. p. 09-30.

PALM, R. C. M. Oncologia. In: CAVALCANTI, A.; GALVÃO, C. Terapia Ocupacional: Fundamentação e Prática. Rio de Janeiro: Editora Guanabara Koogan, 2007. p. 487-492.

PAPASTAVROU, E. et al. Caring for a relative with dementia: family caregiver burden. Journal of Advanced Nursing, Oxford, v. 58, n. 5, p. 446-457, June 2007. http://dx.doi.org/10.1111/j.1365-2648.2007.04250.x

PEDRO, K. S.; MARCON, S. S. Perfil e vivência dos cuidadores informais de doentes crônicos assistidos pelo NEPAAF - Núcleo de estudos, pesquisa, assistência e apoio à família. Online Brazilian Journal of Nursing, Niterói, v. 6, Jan 2007. [aproximadamente 10 p.]. Disponível em: <http://www.objnursing.uff.br/index.php/nursing/article/ view/653/153>. Acesso em: 20 set. 2010.
PEREIRA, L. L.; DIAS, A. C. G. O familiar cuidador do paciente terminal: processo de despedida no contexto hospitalar. Psico, Porto Alegre, v. 38, n. 1, p. 55-65, jan./ abr. 2007.

PY, L. Cuidar do cuidador: transbordamento e carência. Revista Brasileira de Cancerologia, Rio de Janeiro, v. 50, n. 4, p. 346-350, 2004

QUEIROZ, M. S. Saúde e doença: um enfoque antropológico. São Paulo: EdUSC, 2003.

REZENDE, V. et al. Depressão e ansiedade nos cuidadores de mulheres em fase terminal de câncer de mama e ginecológico. Revista Brasileira de Ginecologia e Obstetrícia, Rio de Janeiro, v. 27, n. 12, p. 737-743, dez. 2005. http:// dx.doi.org/10.1590/S0100-72032005001200006

SALES, C. A.; MATOS, P. C. B.; MENDONÇA, D. P. R.; MARCON, S. S. Cuidar de um familiar com câncer: o impacto no cotidiano de vida do cuidador. Revista Eletrônica de Enfermagem, Goiânia, v. 12, n. 4, p. 616-621, 2010.

SILVA, C. A. L. O. et al. Prevalência de Fatores Associados ao Câncer entre Alunos de Graduação nas Áreas da Saúde e Ciências Biológicas. Revista Brasileira de Cancerologia, Rio de Janeiro, v. 56, n. 2, p. 243-249, 2010.

SPINK, M. J. P. A. Construção Social do Saber sobre a Saúde e a Doença. In: SPINK, M. J. P. A. Psicologia Social e Saúde: práticas, saberes e sentidos. Petrópolis: Editora Vozes, 2004. p. 40-60.

TURATO, E. R. Tratado da Metodologia da Pesquisa Clínico-Quantitativa. São Paulo: Vozes, 2003.

VALLE, E. R. M. Psico-oncologia pediátrica: fé e esperança como recursos existenciais. In: ANGERAMI-CAMON, V. A. (Org.). Vanguarda em Psicoterapia FenomenológicaExistencial. São Paulo: Ed. Thomson, 2004. p. 27-40.

ZAGO, M. M. F. et al. O adoecimento pelo câncer de laringe. Revista da Escola de Enfermagem da USP, São Paulo, v. 35, n. 2, p. 108-114, jun. 2001. http://dx.doi. org/10.1590/S0080-62342001000200003

WANDERBROOCKE, A. C. N. S. Perfil do cuidador do paciente idoso com câncer. Psico, Porto Alegre, v. 33, n. 2, p. 401-412, jul./dez. 2002

WANDERBROOCKE, A. C. N. S. Cuidando de um familiar com câncer. Psicologia Argumento, Curitiba, v. 23 , n. 41 , p. 17-23, 2005.

\section{Contribuição dos Autores}

Mariana Ortelani de Toledo: Responsável pelo desenvolvimento da pesquisa e concepção e revisão do artigo. Maria Luisa Gazabim Simóes Ballarin: Responsável pela orientação da pesquisa, concepção e revisão do artigo.

\section{Notas}

${ }^{1}$ Alguns dos resultados deste estudo foram apresentados no $11^{\circ}$ Congresso Brasileiro de Psico-Oncologia e IV Encontro Internacional de Cuidados Paliativos em Oncologia, como tema livre, em setembro de 2010. 